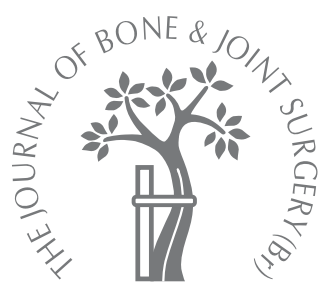

\title{
A histological study of articular cartilage after rotational acetabular osteotomy for hip dysplasia
}

K. Shimogaki, Y. Yasunaga, M. Ochi

From Miyoshi Central Hospital, Miyoshi, Japan
K. Shimogaki, MD, Orthopaedic Surgeon Department of Orthopaedic Surgery

Miyoshi Central Hospital, 531 Higashisakeya, Miyoshi, Hiroshima 728-8502, Japan.

Y. Yasunaga, MD Associate Professo M. Ochi, MD, Professor and Chairman

Department of Orthopaedic Surgery

Graduate School of Biomechanical Studies, Hiroshima University, 1-2-3 Kasumi, Minami-ku, Hiroshima 734-8551, Japan.

Correspondence should be sent to Dr K. Shimogaki; e-mail: koichishimogaki@ yahoo.co.jp

(C)2005 British Editorial Society of Bone and Joint Surgery doi:10.1302/0301-620X.87B7. $15589 \$ 2.00$

$J$ Bone Joint Surg $[B r]$ 2005;87-B:1019-23.

Received 20 April 2004;

Accepted after revision

8 October 2004

\begin{abstract}
Acetabular dysplasia was produced in $\mathbf{2 4}$ immature white rabbits. A rotational acetabular osteotomy was then carried out and radiological and histological studies of the articular cartilage were made.

In the hips which did not undergo osteotomy, radiographs at 26 weeks showed that residual subluxation remained and arthritic changes such as narrowing of the joint space or dislocation were still seen. However, in the operated group there was a remarkable increase in cover, but arthritic changes were not observed. After 24 weeks, the Mankin grading score in the operated group was significantly lower than that in the non-operated group. The latter hips showed an irregular surface of the cartilage, exfoliation and proliferation of synovial tissue. In those undergoing osteotomy, primary cloning of chondrocytes or hypercellularity was seen and at $\mathbf{2 4}$ weeks after operation and metaplasia of the cartilage in the fibrous tissue was observed in the boundary between the medial area of the acetabulum and the acetabular fossa.
\end{abstract}

Periacetabular osteotomy including rotational acetabular osteotomy (RAO), ${ }^{1}$ triple osteotomy, ${ }^{2}$ spherical acetabular osteotomy, ${ }^{3}$ and dial osteotomy ${ }^{4}$ now occupy an important position in the surgical treatment of acetabular dysplasia in young patients. In a previous study we performed experiments in normal rabbits using RAO and described the changes in the articular cartilage induced by a post-operative increase in the load-bearing stress. ${ }^{5}$ We speculated that the results reflected the postoperative histological changes seen in the weight-bearing part of the acetabulum in response to clinically minimal acetabular dysplasia. In order to study the changes in the articular cartilage after RAO in clinically moderate and severe acetabular dysplasia, we produced acetabular dysplasia in rabbits and then carried out periacetabular osteotomy after which we examined the post-operative histological changes in the articular cartilage. The study was approved by the ethics committee of Hiroshima University.

\section{Materials and Methods}

We produced acetabular dysplasia in 24 immature white rabbits (four-week-olds) using the procedure described by Michelsson and Langenskiold. ${ }^{6}$ Under an intravenous infusion of Nembutal of $60 \mathrm{mg} / \mathrm{kg}$, a Kirschner-wire (K-) was inserted from the anterolateral aspect of the lower end of the left tibia to the proximal end of the femur and the knee fixed in extension. The right hip was left intact. Between nine and 14 days after fixation the left hip showed radiological evidence of acetabular dysplasia with lateralisation of the femoral head. The K-wire was then removed and the knee mobilised.

Two weeks later a periacetabular osteotomy was carried out under an intravenous infusion of Nembutal. A curved incision was centred on the apex of the greater trochanter, gluteus medius was separated from the greater trochanter and the bases of the ilium, ischium and pubic bones were exposed. Osteotomies were made at the base of these bones, and the acetabulum was rotated laterally with the ilioischial axis as the centre and then fixed with a K-wire. Gluteus medius was sutured to the greater trochanter and the wound closed (Fig. 1). ${ }^{5}$ No external fixation was used and the animals were allowed to mobilise after the operation.

Four groups of animals were assessed. Of those undergoing acetabular osteotomy, six were killed by massive doses of Nembutal 12 weeks after operation (group A) and six after 24 weeks (group B). In those not having an osteotomy six were killed at 14 weeks after removal of the K-wire (group C) and six 26 weeks after removal of the wire (group D). 


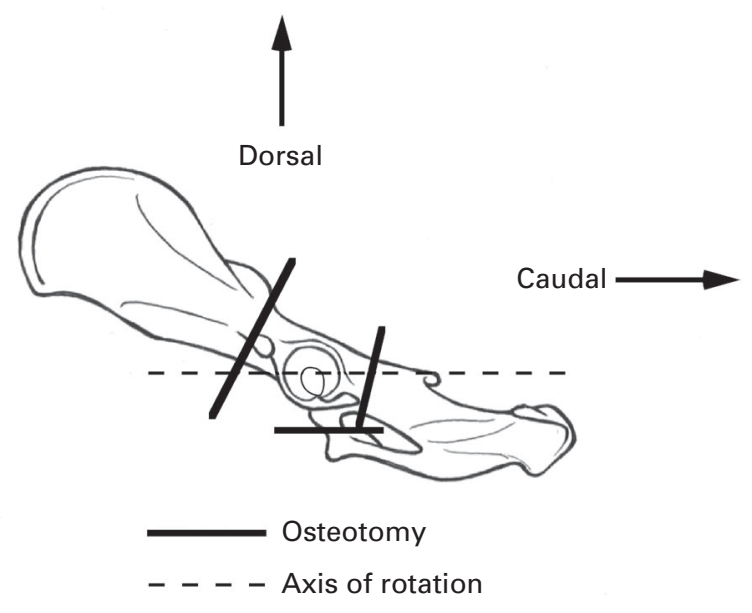

Fig. 1

Diagram of rotational acetabular osteotomy in the rabbit model illustrating the osteotomies and the axis of rotation used (reproduced with permission from Springer Science and Business Media: Yasunaga Y, Ochi M, Ikuta Y, Shimogaki K, Dohi D. Rotational acetabular osteotomies: a rabbit model. Arch Orthop Trauma Surg 1997;116:74-6).

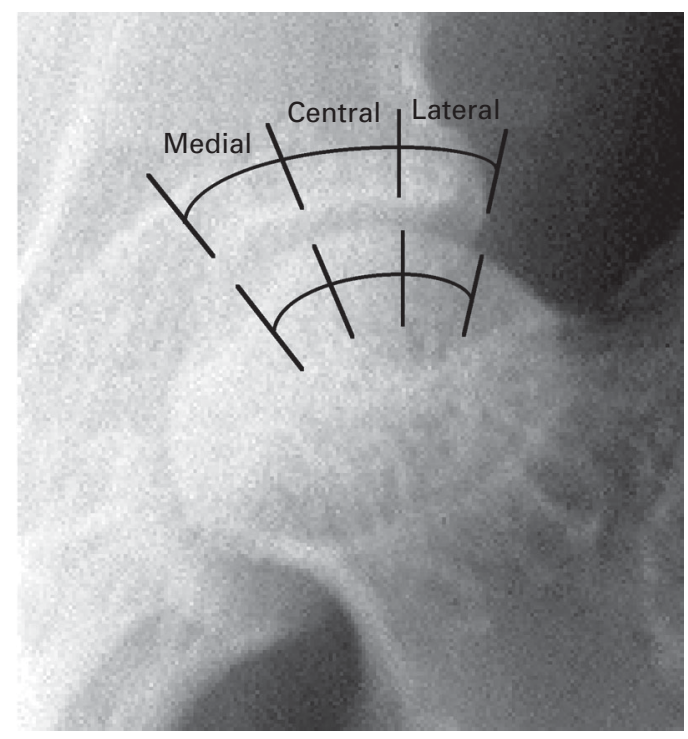

Fig. 2

Diagram showing the areas of the acetabulum and femoral head examined histologically.

Anteroposterior radiographs of the pelvis were obtained at the time of insertion and removal of the K-wire, immediately before and after acetabular osteotomy and at the time of collection of the specimens. The centre-edge (CE) angle ${ }^{7}$ and the acetabular roof obliquity $(\mathrm{AC} \text { angle })^{8}$ were measured and the presence or absence of arthritic changes noted.

After preparing histological specimens from areas perpendicular to the weight-bearing site in the ilium, and staining with haemotoxylin and eosin and Safranin-O, the articular cartilage was assessed using the grading system

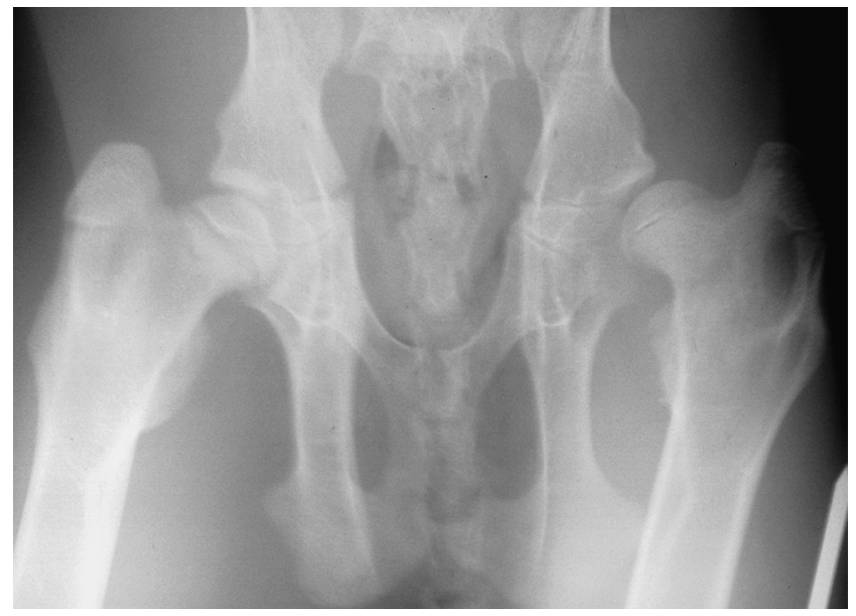

Fig. 3

Radiograph of the hips 14 days after immobilisation of the left knee in the fully extended position by a K-wire. Acetabular dysplasia and lateralisation of the femoral head are evident in the left hip.

Table I. Median (range) values of the centre-edge angle $\left({ }^{\circ}\right)$ for all groups at the various time intervals

\begin{tabular}{|c|c|c|c|}
\hline Group & $\begin{array}{l}\text { Before insertion } \\
\text { of K-wire }\end{array}$ & $\begin{array}{l}\text { Two weeks after } \\
\text { K-wire removal }\end{array}$ & $\begin{array}{l}\text { At collection of } \\
\text { specimens }\end{array}$ \\
\hline A & $10 \quad(7.0 \text { to } 12)^{*}$ & $4.0(-11 \text { to } 8.0)^{* \dagger}$ & $(27 \text { to } 40)^{\text {tई }}$ \\
\hline B & $9.5(5.0 \text { to } 11)^{*}$ & $-1.0(-13 \text { to } 9.0)^{* \dagger}$ & $(30 \text { to } 45)^{\dagger \pi}$ \\
\hline C & $10 \quad(8.0 \text { to } 13)^{*}$ & $6.0(-11 \text { to } 8.0)^{* \neq}$ & $3.0(-7.0 \text { to } 6.0)^{\ddagger \S}$ \\
\hline D & $9.0(8.0 \text { to } 10)^{*}$ & $7.0(-18 \text { to } 8.0)^{* f}$ & $-3.0(-9.0 \text { to } 5.0)^{\neq \pi}$ \\
\hline Overall & $10 \quad(5.0 \text { to } 13)^{*}$ & $4.0(-18 \text { to } 9.0)^{*}$ & \\
\hline
\end{tabular}

* $\mathrm{p}<0.05$, before insertion compared with two weeks after $\mathrm{K}$-wire removal $\dagger p<0.01$, two weeks after K-wire removal compared with value at collection of specimen

$\neq \mathrm{p}<0.05$, two weeks after K-wire removal compared with value at collection of specimen

$\S p<0.01$, groups $A$ and $C$ compared

I $p<0.01$, groups $B$ and $D$ compared

Table II. Median (range) values for the acetabular roof obliquity angle $\left(^{\circ}\right.$ ) for all groups at the various time intervals

\begin{tabular}{|c|c|c|c|}
\hline Group & $\begin{array}{l}\text { Before insertion } \\
\text { of K-wire }\end{array}$ & $\begin{array}{l}\text { Two weeks after } \\
\text { K-wire removal }\end{array}$ & $\begin{array}{l}\text { At collection of } \\
\text { specimens }\end{array}$ \\
\hline $\bar{A}$ & $20(13 \text { to } 23)^{*}$ & $27(17 \text { to } 35)^{* \dagger}$ & $3.0(-1.0 \text { to } 9.0)^{\dagger \S}$ \\
\hline B & $16(13 \text { to } 24)^{*}$ & $33(26 \text { to } 34)^{* \dagger}$ & $1.5(-4.0 \text { to } 9.0)^{\dagger \uparrow}$ \\
\hline C & $21(13 \text { to } 25)^{*}$ & $33(19 \text { to } 36)^{* \neq}$ & $34(27 \text { to } 40)^{\dagger \S}$ \\
\hline D & $17(14 \text { to } 20)^{*}$ & $23(21 \text { to } 36)^{* \neq}$ & $41 \quad(30 \text { to } 45)^{\dagger \uparrow}$ \\
\hline Overall & $19(13 \text { to } 25)^{*}$ & $31(17 \text { to } 36)^{*}$ & \\
\hline
\end{tabular}

${ }^{*} \mathrm{p}<0.05$, before insertion compared with two weeks after K-wire removal $+p<0.01$, two weeks after K-wire removal compared with value at collection of specimen

$\ddagger p<0.05$, two weeks after K-wire removal compared with value at collection of specimen

$\S p<0.01$, groups $A$ and $C$ compared

If $p<0.01$, groups $B$ and $D$ compared

described by Mankin et al. ${ }^{9}$ This system scores the arthritic changes in the cartilage, including the structure, the cells, Safranin-O staining and the integrity of the tidemark. The score ranged from 0 to 14 with 0 indicating normal articu- 


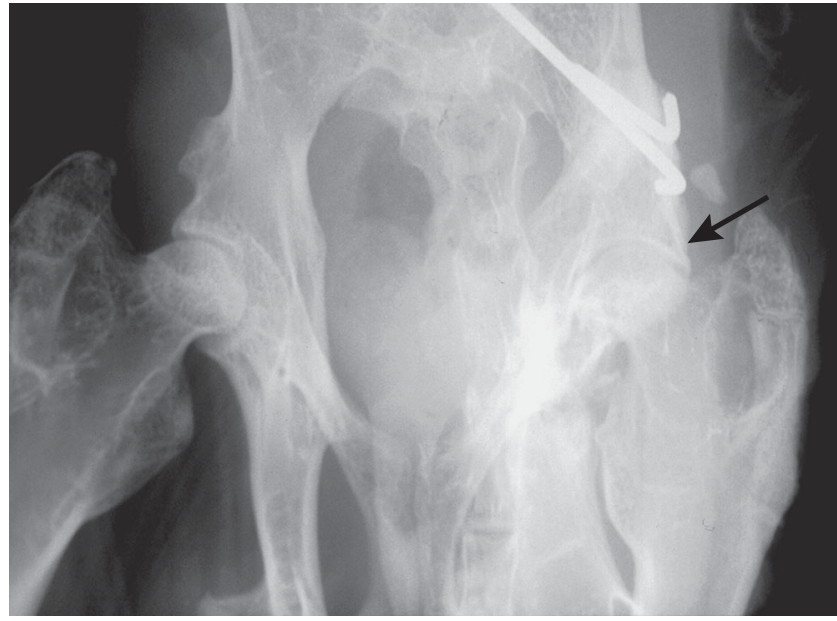

Fig. 4

Radiograph of a hip (group B). The centre-edge and acetabular roof of obliquity angles showed a marked increase in cover compared with before operation.

lar cartilage. Assessment was made of the medial, central and lateral parts of the primary weight-bearing area of the acetabulum. The surfaces of the femoral head were also observed as well as the corresponding three areas of the acetabulum (Fig. 2).

In groups A and B immunohistochemical staining ${ }^{5}$ was performed using three types of monoclonal antibody for proteoglycan (anti-4 sulphated chondroitin proteoglycan, 2-B-6, anti-6 sulphated chondroitin proteoglycan, 3-B-3; and anti-dermatan sulphate proteoglycan, 6-B-6; Seikagaku, Tokyo, Japan).

Statistical analysis. We used the two-way analysis of variance (ANOVA). The level of significance was recorded as $\mathrm{p}<0.05$.

\section{Results}

Radiological evaluation. Two weeks after removal of the $\mathrm{K}$-wire the median $\mathrm{CE}$ angle was $4.0^{\circ}\left(-18^{\circ}\right.$ to $\left.9.0^{\circ}\right)$ and the median AC angle $31^{\circ}\left(17^{\circ}\right.$ to $\left.36^{\circ}\right)$. Acetabular dysplasia, lateralisation of the femoral head and subluxation were apparent (Fig. 3; Tables I and II). In the operated groups, the $\mathrm{CE}$ angle (median $3^{\circ},-9^{\circ}$ to $10^{\circ}$ ) and the $\mathrm{AC}$ angle (median $26^{\circ}, 16^{\circ}$ to $33^{\circ}$ ) at the time of removal of the K-wire showed no significant difference from those seen at two weeks after removal of the wire immediately before the acetabular osteotomy.

At the time of the collection of specimens in the operated groups, the $\mathrm{CE}$ and the $\mathrm{AC}$ angles showed a remarkable increase in cover when compared with those at two weeks after removal of the K-wire (Fig. 4; Tables I and II). By contrast, in those which did not undergo osteotomy, acetabular dysplasia, lateralisation of the femoral head and residual subluxation were observed, and in two cases in group D dislocation had occurred. In the operated groups the $\mathrm{CE}$ (median $36^{\circ}, 29^{\circ}$ to $45^{\circ}$ ) and the AC angles (median $3.0^{\circ}$,
Table III. Median (range) values for the Mankin grading score ${ }^{9}$ for the acetabular cartilage for all areas and all groups

\begin{tabular}{lllll}
\hline Group & Medial & Central & Lateral & Overall \\
\hline A & $6.0(4 \text { to } 8)^{* \dagger}$ & $4.5(2 \text { to } 5)^{*}$ & $3.5(1 \text { to } 5)^{\dagger}$ & $4.5(1$ to 8$)$ \\
B & $4.0(3 \text { to } 5)^{* \ddagger}$ & $3.0(2 \text { to } 4)^{*}$ & $3.0(2 \text { to } 4)^{\ddagger}$ & $3.5(2 \text { to } 5)^{\dagger}$ \\
C & $5.5(3$ to 6$)$ & $4.0(3$ to 5$)$ & $6.0(4$ to 7$)$ & $4.5(3$ to 7$)$ \\
D & $5.5(2$ to 8$)$ & $5.5(4$ to 7$)$ & $6.5(3$ to 9$)$ & $6.0(2 \text { to } 9)^{\dagger}$ \\
\hline
\end{tabular}

* $\mathrm{p}<0.05$, medial and central compared; groups A and B compared $\dagger p<0.01$, medial and lateral compared; groups $B$ and D compared $\ddagger p<0.05$, medial and lateral compared

Table IV. Median (range) values for the Mankin grading score ${ }^{9}$ for the cartilage of the femoral head for all areas and all groups

\begin{tabular}{lllll}
\hline Group & Medial & Central & Lateral & Overall \\
\hline A & $3.0(2$ to 4$)$ & $4.0(3$ to 6$)$ & $4.5(3$ to 6$)$ & $4.0(2 \text { to } 6)^{*}$ \\
B & $2.0(0 \text { to } 4)^{\dagger}$ & $3.0(2 \text { to } 4)^{*}$ & $2.0(1 \text { to } 6)^{\dagger}$ & $2.0(0 \text { to } 6)^{* \dagger}$ \\
C $^{\dagger}$ & $5.0(2$ to 8$)$ & $4.5(2$ to 8$)$ & $5.5(4$ to 7$)$ & $5.0(2$ to 8$)$ \\
D & $6.0(3 \text { to } 9)^{\dagger}$ & $6.0(3 \text { to } 9)^{\dagger}$ & $7.0(5 \text { to } 9)^{\dagger}$ & $6.5{\text { (3 to } 9)^{\dagger}}$
\end{tabular}

* $\mathrm{p}<0.05$, groups A and B compared

$\dagger p<0.01$, groups $B$ and $D$ compared

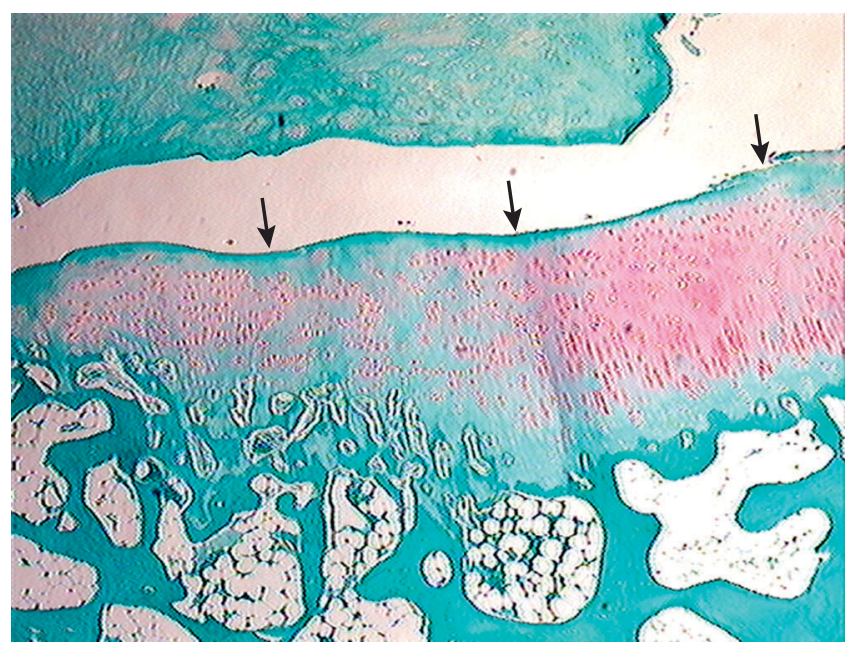

Fig. 5

Photomicrograph of a hip (group D). The cartilage has an irregular surface with exfoliation of the femoral head (Safranin-O, x40)

$-8.0^{\circ}$ to $7.0^{\circ}$ ) immediately after osteotomy showed no significant difference from those at the time of collection of the specimens.

When the two cases of dislocation were excluded, one example of narrowing of the joint space was observed in each of groups $\mathrm{C}$ and $\mathrm{D}$ and deformation of femoral head in one hip in group C and two in group D. However, in the operated groups, necrosis of the acetabulum or the femoral head and narrowing of the joint space were not seen.

Histological evaluation. The two hips which were dislocated in group D were not subjected to histological evaluation of the articular cartilage.

The acetabulum (Table III). When each area was compared in group A and group B, the scores in the medial portion 


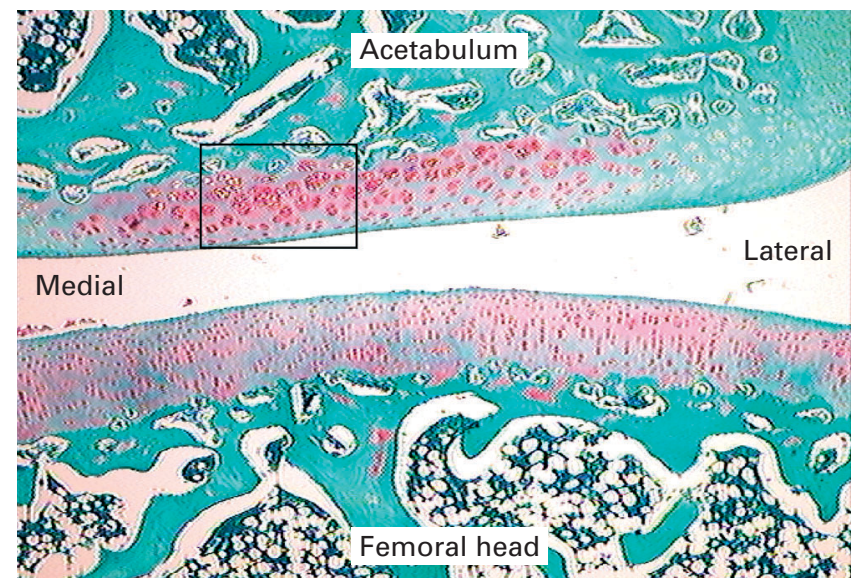

Fig. $6 a$

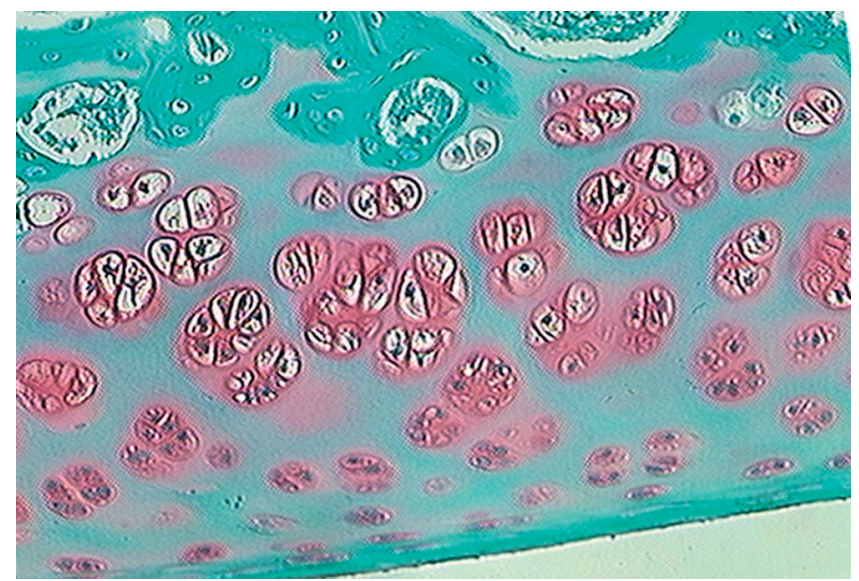

Fig. $6 \mathrm{~b}$

Photomicrographs of a hip (group A). There is hypercellularity of cartilaginous cells on the medial area of the acetabulum (Safranin-O stain) a) $\mathrm{x} 40$; b) x200.

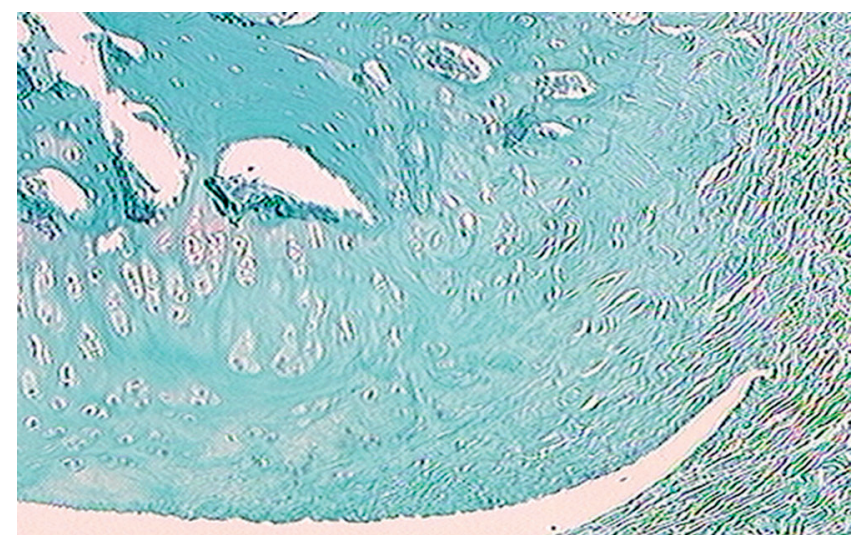

Fig. 7 a

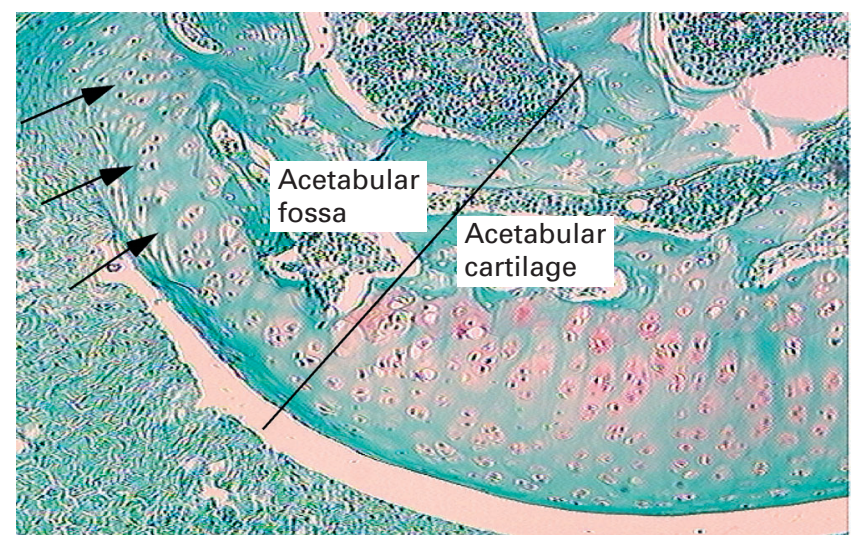

Fig. $7 \mathrm{~b}$

Photomicrographs of the medial area of the acetabulum (group B) showing a) the normal and b) the affected sides. The arrows indicate cartilaginous metaplasia which was observed in the tissue at the boundary (see line) between the medial area of the acetabulum and the acetabular fossa on the affected side (Safranin-0, x100).

were significantly higher compared with those in the central and the lateral areas. The grading score in the medial area of group B was significantly decreased compared with that in group A. The score was significantly higher in group D than in group $\mathrm{B}$.

Femoral head (Table IV). In those undergoing osteotomy, the score in group A was significantly higher than that in group B. Considering all the groups at 24 weeks the grading scores in all parts were significantly higher in group D than in group B.

On both the acetabular and femoral sides, the principal changes in the non-operated groups were an irregular surface of the cartilage, exfoliation, and proliferation of synovial tissue (Fig. 5). In those undergoing osteotomy cloning of cartilaginous cells or hypercellularity was prominent (Fig. 6).
In two of the six hips in group B, metaplasia of the cartilage was observed in the tissue at the boundary between the medial area of the acetabulum and the acetabular fossa (Fig. 7).

Immunohistological evaluation. Immunohistological staining in groups $\mathrm{A}$ and $\mathrm{B}$ was positive for monoclonal antibodies 2-B-6 and 3-B-3 in the cartilage of the acetabulum and the femoral head. No difference was observed between the normal side and the operated side. Staining with 6-B-6, which demonstrates degenerated proteoglycan, was not observed.

\section{Discussion}

In experimental acetabular dysplasia as described by Michelsson and Langenskiold ${ }^{6}$ the knee is fixed in extension and lateralisation of the femoral head is sought by enhancing 
the tension of the hamstrings. According to Yamamoto, ${ }^{10}$ after fixation of the knee for one week, the acetabular dysplasia is reversible, but after two weeks the dysplasia remains and the histological changes in the cartilaginous cells become irreversible. In our model, the period of fixation ranged from nine days to two weeks, and in all cases acetabular dysplasia was apparent in comparison with the control side. Similar changes remained two weeks after removal of the wire. Between 14 and 26 weeks after removal of the wire the changes progressed leading to narrowing of the joint space or deformity of the femoral head with some hips progressing to complete dislocation. These findings coincide with those of Yamamoto. ${ }^{10}$

In the hips which did not undergo osteotomy the main changes seen were exfoliation of the articular cartilage, an irregular joint surface and rarefaction, all of which progressed with time. In those undergoing operation histological examination showed cloning and increased stain uptake of the cartilage matrix which had decreased by 24 weeks with absence of the changes characteristic of osteoarthritis. The clones of chondrocytes observed in the operated groups were considered to represent the synthetic clones noted by Mitchell, Lee and Shepard. ${ }^{11}$ The remodelling of the cartilage tissue responding to the changes in the dynamic environment because of displacement of the hip in the early post-operative period develops at the site corresponding to the new weight-bearing stress and becomes stable with time.

Raab, Lohr and Krauspe, ${ }^{12}$ after inducing dislocation of the hip in rabbits conducted a histological study on the acetabular weight-bearing cartilage in a group which was reduced early and in another which was left dislocated. In the untreated dislocation group the hyaline cartilage decreased towards the weight-bearing area and cartilaginous cells showed a tendency for metaplasia to fibrocartilage. However, in the group which was reduced early similar changes were reversed by restoration of weightbearing stress after reduction. The earlier the reduction occurred, the more complete the restoration to normal. It therefore appears that the application of an appropriate amount of loading, while the articular cartilage still maintains the potential for remodelling, will restore the integrity of the cartilage at the site of weight-bearing.

In 1992 Hiranuma et $a l^{13}$ carried out a histological study after a Chiari osteotomy and noted that cartilaginous metaplasia resembling fibrocartilage developed in the joint capsule at the acetabulum, which became the new weightbearing surface. We observed cartilaginous metaplasia at the boundary between the medial area of the acetabulum and the acetabular fossa, which became the new weightbearing surface. When comparing the histological appearances with those of the opposite normal hip, the displacement site of weight-bearing in the acetabular fossa was found to correspond to the area where fibrous tissue was present near the attachment of the ligamentum teres. It seems that metaplasia had developed in response to changes in the dynamic environment because of displacement of the non-cartilaginous tissue to the acetabular fossa. Clinically, the weight-bearing surface enlarged because of formation of subchondral bone, which may be related to this cartilaginous metaplasia. ${ }^{14}$

Our study suggests that RAO in moderate to severe acetabular dysplasia, which in itself does not cause arthritic changes, will allow remodelling of cartilaginous tissue corresponding to the degree of displacement. This may prevent arthritic changes. However, as described by Yasunaga et $\mathrm{al},{ }^{15}$ the magnitude of damage to the cartilage before RAO is undertaken influences the progression of post-operative arthritis. Unless there is favourable remodelling, degeneration of the cartilage may be induced. Thus, in order to obtain favourable results it is necessary to have conditions which will favour remodelling. Hence, when degenerative changes are advanced and for relatively old patients in whom the activity of cartilage may be reduced, the indications for this procedure must be carefully considered.

No benefits in any form have been received or will be received from a commercial party related directly or indirectly to the subject of this article.

\section{References}

1. Ninomiya S, Tagawa H. Rotational acetabular osteotomy for the dysplastic hip. J Bone Joint Surg [Am] 1984;66-A:430-6.

2. Steel HH. Triple osteotomy of the innominate bone. J Bone Joint Surg [Am] 1973; 55-A:343-50.

3. Wagner H. Experiences with spherical acetabular osteotomy for the correction of the dysplastic acetabulum. In: Progress in orthopaedic surgery 2: acetabular dysplasia, skeletal dysplasia in childhood. Berlin: Springer, 1978:131-45

4. Eppright RH. Dial osteotomy of the acetabulum in the treatment of dysplasia of the hip. J Bone Joint Surg [Am] 1975;57-A:1172.

5. Yasunaga Y, Ochi M, Ikuta Y, Shimogaki K, Dohi D. Rotational acetabular osteotomies: a rabbit model. Arch Orthop Trauma Surg 1997;116:74-6.

6. Michelsson JE, Langenskiold A. Dislocation or subluxation of the hip: regular sequels of immobilization of the knee in extension of young rabbits. J Bone Joint Surg [Am] 1972;54-A:1177-86

7. Wiberg G. Studies on dysplastic acetabula and congenital subluxation of the hip joint: with special reference to the complication of osteoarthritis. Acta Chir Scand 1939;83 (Suppl 58):1-134.

8. Massie WK, Howorth MB. Congenital dislocation of the hip: part I: method of grading results. J Bone Joint Surg [Am] 1950;32-A:519-31.

9. Mankin HJ, Dorfman H, Lippiello L, Zarnis A. Biochemical and metabolic abnormalities in articular cartilage from osteo-arthritic human hips: Il: correlation of morphology with biochemical and metabolic data. J Bone Joint Surg [Am] 1971:53-A: 523-7.

10. Yamamoto $\mathbf{N}$. Changes of the acetabular cartilage following experimental subluxation of the hip joint in rabbits. Nippon Sekeigeka Gakkai Zasshi 1983;57:1741-53 (in Japanese).

11. Mitchell N, Lee ER, Shepard N. The clones of osteoarthritic cartilage. J Bone Joint Surg [Br] 1992; 74-B:33-8.

12. Raab P, Lohr J, Krauspe R. Remodeling of the acetabulum after experimental hip joint dislocation: an animal experiment study of the rabbit. Z Orthop Ihre Grenzgeb 1998;136:519-24 (in German).

13. Hiranuma S, Higuchi F, Inoue A, Miyazaki M. Changes in the interposed capsule after Chiari osteotomy: an experimental study on rabbits with acetabular dysplasia J Bone Joint Surg [Br] 1992;74-B:463-7.

14. Yasunaga Y, Ikuta Y, Shigenobu T, et al. Rotational acetabular osteotomy for hip dysplasia: spontaneous medial enlargement of the acetabulum. Acta Orthop Scand 2001;72:8-12

15. Yasunaga Y, Ikuta Y, Kanazawa T, Takahashi K, Hisatome T. The state of the articular cartilage at the time of surgery as an indication for rotational acetabular osteotomy. J Bone Joint Surg [Br]2001;83-B:1001-4. 